\title{
Prognostic value of inflammation-based scores in patients receiving radical resection for colorectal cancer
}

\author{
Fang Wang ${ }^{1,2+}$, Wenzhuo $\mathrm{He}^{3 \dagger}$, Chang Jiang ${ }^{3}$, Guifang Guo ${ }^{3}$, Bin Ke ${ }^{4}$, Qiangsheng Dai ${ }^{2}$, Jianting Long ${ }^{2 *}$ \\ and Liangping $\mathrm{Xia}^{3^{*}}$
}

\begin{abstract}
Background: The modified Glasgow Prognostic Score (mGPS) and the neutrophil-to-lymphocyte ratio (NLR) are conventional inflammation-based scores for colorectal cancer (CRC). The systemic inflammation score (SIS) has been shown to be more informative than the mGPS in CRC. The albumin-NLR, composed of albumin and the NLR, can also be a candidate for a valuable inflammation score. However, about the utility of the MGPS, SIS, and albuminNLR for CRC patients who have received radical resections remains unclear.
\end{abstract}

Methods: This study enrolled 877 CRC patients, who underwent radical surgical resection between January 1, 2007 and December 31, 2014. The prognostic values of the mGPS, SIS, and albumin-NLR were compared by the KaplanMeier survival analysis, multivariate Cox regression modelling, and the time-dependent receiver operating characteristic curve analysis (ROC).

Results: In the Kaplan-Meier analysis, all three inflammation scores were significantly associated with overall survival $(\mathrm{OS})$ in the group including all the patients (mGPS, $p=0.016$; SIS, $p<0.001$; albumin-NLR, $p=0.007$ ) and in the leftsided colon tumour subgroup (mGPS, $p=0.029$; SIS $p=0.0013$; albumin-NLR, $p=0.001$ ). In the right-sided colon tumour subgroup, only the albumin-NLR was associated with OS ( $p=0.048)$. The albumin-NLR was the only independent prognostic factor of the three scores for OS in the multivariate survival analysis.

Conclusions: The albumin-NLR outperformed both the SIS and mGPS in predicting OS in CRC patients undergoing radical resection.

Keywords: Colorectal cancer, Radical resection, Modified-Glasgow prognostic score, Systemic inflammation score, Albumin-neutrophil-to-lymphocyte ratio, And intratumoural chronic inflammatory cell

\section{Background}

Colorectal cancer is the leading cause of cancer mortality worldwide [1]. Despite immense efforts in developing advanced treatments for this disease, the overall survival for colorectal cancer remains poor, even in patients who receive resection with curative intent, with only $50 \%$ of patients surviving 5 years post-surgery [2].

\footnotetext{
*Correspondence: longjianting@163.com; xialiangping@163.com

${ }^{\dagger}$ Fang Wang and Wenzhuo He contributed equally to this work.

${ }^{2}$ Department of Oncology, The First Affiliated Hospital of Sun Yat-sen

University, 58, the 2nd Zhongshan Road, Guangzhou 510080, China

${ }^{3}$ Sun Yat-sen University Cancer Center; State Key Laboratory of Oncology in

South China; Collaborative Innovation Center for Cancer Medicine, 651,

Dongfengdong Road, Guangzhou 510060, China

Full list of author information is available at the end of the article
}

As commonly recognized, the progression of colorectal tumours is dependent not only on the tumour characteristics alone but also on the systemic environment of the host. Furthermore, there is increasing evidence that both local and systemic inflammatory responses play an important role in the progression of a variety of common solid tumours [3-7].

For systemic inflammation indices, the neutrophilto-lymphocyte ratio (NLR) and the lymphocyte-to-monocyte ratio (LMR) have been identified as prognostic markers for colorectal cancer [8-10]. Furthermore, the modified Glasgow Prognostic Score (mGPS), which comprises the serum C-reactive protein (CRP) and serum albumin levels, has been demonstrated as a

(c) The Author(s). 2018 Open Access This article is distributed under the terms of the Creative Commons Attribution 4.0 International License (http://creativecommons.org/licenses/by/4.0/), which permits unrestricted use, distribution, and 
favourable prognostic index for colorectal cancer (CRC) patients [11-13]. Recently, Suzuki et al. developed the systemic inflammation score (SIS), which comprises LMR and serum albumin levels and was found to be superior to the mGPS in predicting prognosis for colorectal cancer patients [14].

For local inflammation factors, tumour-infiltrating lymphocytes (TILs) have been identified as robust factors predicting outcomes in several reports investigating solid tumours [15-18]. In 1986, Jass first proposed TIL as a novel independent prognostic factor for CRC, and this new system was considered superior to the Duke's staging system [19]. However, the prognostic value of TILs in CRC remains controversial due to a limited number of studies. In addition, the correlation of systemic inflammation indices and local inflammation factors has rarely been studied.

The aim of this study was to compare the prognostic value of the mGPS, SIS, and albumin-NLR, comprising serum albumin and NLR, and to determine the relationship between local and systemic inflammation factors.

\section{Methods}

\section{Patient selection}

A series of 877 CRC patients were enrolled in this study. The patients were treated with radical surgical resection at the Sun Yat-Sen University Cancer Center between January 1, 2007, and December 31, 2014. The inclusion criteria were the following: (1) The patient underwent radical resection at Sun Yat-Sen University Cancer Center for pathologically confirmed American Joint Committee on Cancer (AJCC) stage I to III CRC; (2) the patient received routine analyses of blood, CRP, and serum albumin levels before surgery; and, (3) the resected specimens and pathology slides were stored at our institution. The exclusion criteria were the following: (1) the patient received neoadjuvant chemo-radiotherapy or radiation therapy; (2) the patient experienced acute or chronic inflammation; (3) the patient experienced double cancer; and, (4) the patient's case had insufficient data.

A routine follow-up was conducted by the follow-up department of Sun Yat-Sen University Cancer Center. Overall survival (OS) was calculated from the time of diagnosis to the date of death or the last follow-up visit.

\section{Data extraction}

The clinical characteristics and serological examination results of all the study patients were collected from medical records. Hematoxylin and eosin (H\&E) stained tissue sections from surgically resected tumour specimens were reviewed for all CRC patients. One pathologist evaluated all available sections for each patient and selected the slide with the highest intratumoural chronic inflammatory cell (CIC) density. Two pathologists scored the average CIC density (lymphocytes, neutrophils and plasma cells) in both the central region and the invasive margin of the tumour. Follow-up data were available for all patients, and the latest follow-up was conducted on September 29, 2017. The OS was defined as the time from diagnosis to death from any cause or censored at the date of the last follow-up.

\section{Inflammation-based incidences}

The neutrophil-granulocyte cell count, lymphocyte cell count, monocyte cell count, serum albumin, and CRP levels were recorded before treatment. The mGPS score was established using the serum CPR and albumin levels as previously described [20]. The NLR is equal to the neutrophil count divided by the total lymphocyte count; the LMR is derived from the lymphocyte count divided by the monocyte count. The cut-off points for NLR and LMR were 2.39 and 3.80 , respectively, which were derived using the ROC analysis. The SIS, which comprises the LMR and serum albumin, was defined as follows: patients with $\mathrm{LMR} \geq 3.8$ and serum albumin $\geq 39.75 \mathrm{~g} / \mathrm{L}$ were scored as 0 ; patients with LMR $<3.8$ or serum albumin $<39.75 \mathrm{~g} / \mathrm{L}$ were scored as 1 ; and patients with LMR $<3.8$ and serum albumin levels $<39.75 \mathrm{~g} / \mathrm{L}$ were scored as 2 . For the albumin-NLR assessment, patients with serum albumin levels $\geq 39.75 \mathrm{~g} / \mathrm{L}$ and NLR $<2.39$ were assigned a score of 0 ; patients with either hypoalbuminemia $(<39.75 \mathrm{~g} / \mathrm{L})$ or elevated in NLR levels $(\geq 2.39)$ were scored as 1 ; and those with both hypoalbuminemia $(<39.75 \mathrm{~g} / \mathrm{L})$ and an increase in NLR levels $(\geq 2.39)$ were scored as 2 .

The average CIC density was evaluated in the central region and the invasive margin of the tumour. At the invasive margin of the tumour: a score of 0 indicated no increase in inflammatory cells; a score of 1 denoted a mild and patchy increase of inflammatory cells at the invasive margin but no destruction of the invading cancer cell islets by the inflammatory cells; a score of 2 was given when inflammatory cells formed a band-like infiltration at the invasive margin with some destruction of the cancer cell islets; and a score of 3 denoted a very prominent inflammatory reaction, forming a cup-like zone at the invasive margin with frequent and invariably present destruction of the cancer cell islets. A similar scale was used at the central region, a score of 0 indicated absence of a reaction; a score of 1 indicated a weak reaction; a score of 2 indicated a moderate reaction; and a score of 3 indicated a severe increase in each cell type.

A Crohn's-like reaction was defined as a transmural lymphoid reaction. It was scored as 0 (absent), 1+ (mild), $2+$ (moderate), or 3+ (marked). 
All the patients were further divided into either the low-response group or high-response group according to the above inflammation reaction scores. The patients with scores of 0 and 1 were placed in the low-response group, while patients with scores of 2 and 3 were included in the high-response group.

\section{Statistical analysis}

The data were presented as the median values and ranges. The distribution of clinicopathological characteristics according to the different groups was analysed using the Chi-squared or Kruskal-Wallis test, where appropriate. The Kaplan-Meier method and the log-rank test were used to study the impact of different clinical factors on OS. The univariate and multivariate survival analyses had hazard ratios (HRs) calculated using the Cox proportional hazards model. The prognostic ability of the different inflammation scoring methods was compared by generating time-dependent receiver operating characteristic (ROC) curves and by calculating the area under the curve (AUC). The time-dependent ROC curve analysis is used to assess the discriminatory power of continuous markers for time-dependent disease outcomes. It is an extension of the ROC curve and can calculate the AUC and concurrently compare the ROC curves [14]. The sequential AUCs were compared between the mGPS, SIS and albumin-NLR using independent and identically distributed representations [14]. A probability value of $p<0.05$ was defined as statistically significant. All statistical analyses were performed using the SPSS statistical package version 22.0 (IBM Corp., Armonk, NY, USA) and the R studio version 3.4.2 (R Foundation for Statistical Computing, Vienna, Austria).

\section{Results}

\section{Patient characteristics}

A total of 877 patients were included in the final analysis. The median age of all patients was 59 years (range, 1988 years). The distribution of sex was 533 (60.8\%) male patients and $344(39.2 \%)$ female patients. The baseline clinical characteristics and pathological findings of the patients are summarized in Table 1. There were 89 (10.1\%) patients with microsatellite instability (MSI). For the distribution of the TNM stage, 106 (12.1\%) patients were stage I, 505 (57.6\%) patients were stage II, and 266 (57.6\%) patients were stage III. The median OS of patients with CRC was 44.53 months (range: $0.73-123.80$ months). The median follow-up period was 46.73 months (range: $0.73-$ 123.80 months).

\section{Clinicopathological findings}

The relationship between patient clinicopathological characteristics and inflammation-based scores is listed in Table 1. The elevated mGPS, SIS and albumin-NLR scores were significantly associated with an advanced T stage $(p=0.021$, $p=0.026, p=0.021)$, microsatellite instability (MSI) status $(p<0.001, p<0.001, p=0.001)$, and local intratumoural inflammation factors, such as higher neutrophil density in the central region of the tumour (all $p<0.001$ ) and the invasive margin of tumour $(p=0.014, p<0.001$, $p=0.01)$, as well as a Crohn's-like reaction $(p<0.001, p<0.001$, $p=0.013)$. The elevated mGPS score was significantly associated with the male sex $(p=0.024)$, left-sided colon lesions $(p<0.001)$, an advanced disease stage $(p<0.001)$, and a lower number of lymph nodes resected $(p=0.009)$. The increased SIS and albumin-NLR scores correlated with older age (both $p<0.001$ ), left-sided colon lesions $(p=0.049, p<0.001)$, and a higher degree of lymphatic invasion $(p=0.018, p=0.049)$. An increased albumin-NLR score was associated with an advanced disease stage $(p=0.001)$, a higher number of total and negative lymph nodes resected $(p=0.004$ and $p=0.047$, respectively), a higher degree of vascular invasion $(p=0.026)$, and a higher density of lymphocytes in the tumour invasive $\operatorname{margin}(p=0.01)$.

\section{Kaplan-Meier analysis}

The Kaplan-Meier curves for the OS rate were divided into three groups according to the mGPS, SIS, and albumin-NLR scores. The influence of the above three factors on the OS of the study patients is shown in Fig. 1. All three inflammation score types were significantly associated with OS (mGPS, $p=0.016$; SIS, $p<0.001$; albumin-NLR, $p=0.007$ ). It should be noted that the curves of the mGPS overlapped. In the right-sided colon tumour subgroup, only the albumin-NLR was associated with OS $(p=0.048)$, with the survival curves being well separated (Fig. 2). In the left-sided colon tumour subgroup, all three score types were independent factors for OS (mGPS, $p=0.029$; SIS $p=0.0013$; albumin-NLR, $p=0.001$ ) (Fig. 3).

For the local tumour-infiltrating inflammation measure, lymphocytes in the central region of the tumour were significantly associated with OS in the group including all patients and the left-sided colon cancer group (Fig. 4), while lymphocytes in the tumour invasive margin were significant factors for OS in all three groups (Fig. 5). Neutrophils in the central region of the tumour were significant factors for OS in the right-sided colon cancer subgroup $(p=0.042)$. The neutrophil count in the central region and the invasive margin of the tumour, as well as a Crohn's-like reaction, failed to predict OS in the other patient subgroups.

\section{Univariate and multivariate survival analyses}

For the univariate survival analysis, factors such as the mGPS, SIS, and albumin-NLR, age, TNM stage, T stage, $\mathrm{N}$ stage, total number of lymph nodes resected (TLN), 
Table 1 Patients' Clinicopathological Characteristics and Associations with the mGPS, SIS and Albumin-NLR

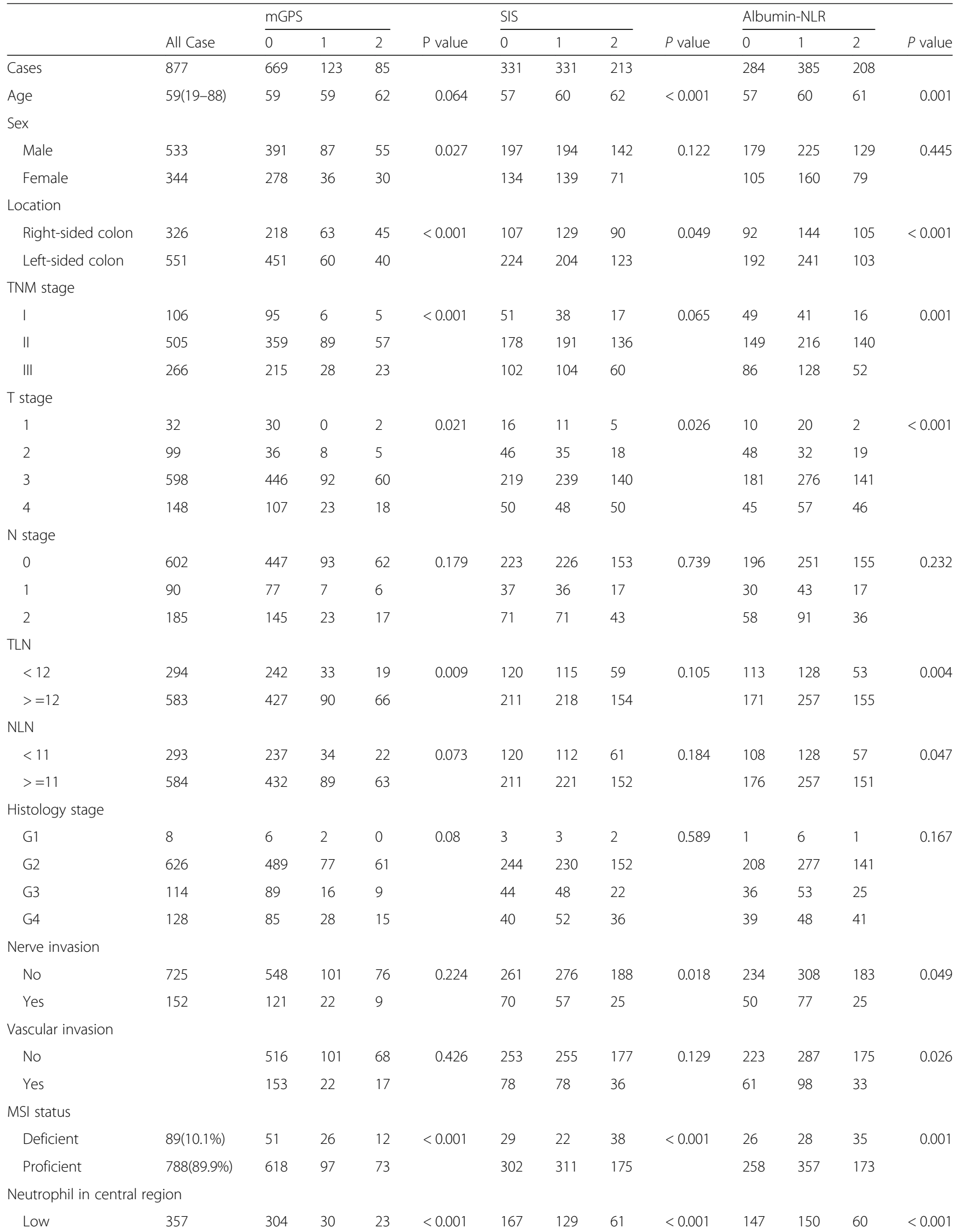


Table 1 Patients' Clinicopathological Characteristics and Associations with the mGPS, SIS and Albumin-NLR (Continued)

\begin{tabular}{|c|c|c|c|c|c|c|c|c|c|c|c|c|c|}
\hline & & $\mathrm{mGP}$ & & & & SIS & & & & Albur & ר-NLR & & \\
\hline & All Case & 0 & 1 & 2 & $P$ value & 0 & 1 & 2 & $P$ value & 0 & 1 & 2 & $P$ value \\
\hline High & 520 & 365 & 93 & 62 & & 164 & 204 & 152 & & 137 & 235 & 148 & \\
\hline Neutrop & largin & & & & & & & & & & & & \\
\hline Low & 561 & 445 & 66 & 50 & 0.014 & 243 & 205 & 113 & $<0.001$ & 200 & 251 & 110 & $<0.001$ \\
\hline High & 316 & 224 & 57 & 35 & & 88 & 128 & 100 & & 84 & 134 & 98 & \\
\hline Lympho & region & & & & & & & & & & & & \\
\hline Low & & 264 & 57 & 28 & 0.142 & 131 & 138 & 80 & 0.661 & 110 & 165 & 74 & 0.203 \\
\hline High & & 405 & 66 & 57 & & 200 & 195 & 133 & & 174 & 220 & 134 & \\
\hline Lympho & margin & & & & & & & & & & & & \\
\hline Low & 427 & 324 & 63 & 40 & 0.809 & 162 & 167 & 98 & 0.636 & 133 & 208 & 86 & 0.01 \\
\hline High & 450 & 345 & 60 & 45 & & 169 & 166 & 115 & & 151 & 177 & 122 & \\
\hline Crohn's- & & & & & & & & & & & & & \\
\hline No & 796 & 624 & 97 & 75 & $<0.001$ & 311 & 301 & 184 & 0.012 & 263 & 355 & 178 & 0.013 \\
\hline Yes & 78 & 43 & 25 & 10 & & 19 & 31 & 28 & & 21 & 28 & 29 & \\
\hline
\end{tabular}

mGPS modified Glasgow Prognostic Score, SIS systemic inflammation score, albumin-NLR albumin-neutrophil-to-lymphocyte ratio, TLN total lymph nodes resected, $N L N$ negative lymph nodes resected, MSI microsatellite instability

number of negative lymph nodes resected (NLN), lymphatic invasion, vascular invasion, lymphocytes in the tumour invasive margin, and lymphocytes in the central region of the tumour, were all identified as significant prognostic factors for OS. All these factors with $p<0.05$ were included in the multivariate survival analysis. The albumin-NLR, age, $\mathrm{N}$ stage, and the total lymph nodes resected (TLN) were demonstrated to be independent prognostic factors for OS (Table 2).

\section{Time-dependent ROC curve analysis}

The time-dependent ROC curve was used to compare the sequential trends of the albumin-NLR, SIS, and mGPS scores, according to the hazard ratios for OS. The time-dependent ROC curve was the integration of the estimated AUC at each time point. In the group including all CRC patients, the time-dependent ROC curve of the albumin-NLR crossed the curves of both the SIS and mGPS at the 15th-month point after surgery and was continuously superior to the other two curves in predicting the 5-year survival rate of patients. Moreover, the ROC curve of the mGPS was superior to the SIS curve. In the right-sided colon cancer subgroup, the albumin-NLR was the most robust inflammation factor for the sequential prediction of OS; at the same time, the time-dependent ROC curve of the mGPS was superior to that of the SIS. For the left-sided colon cancer subgroup, the time-dependent ROC curve of the albumin-NLR was also superior to that of the SIS and mGPS (Fig. 6).
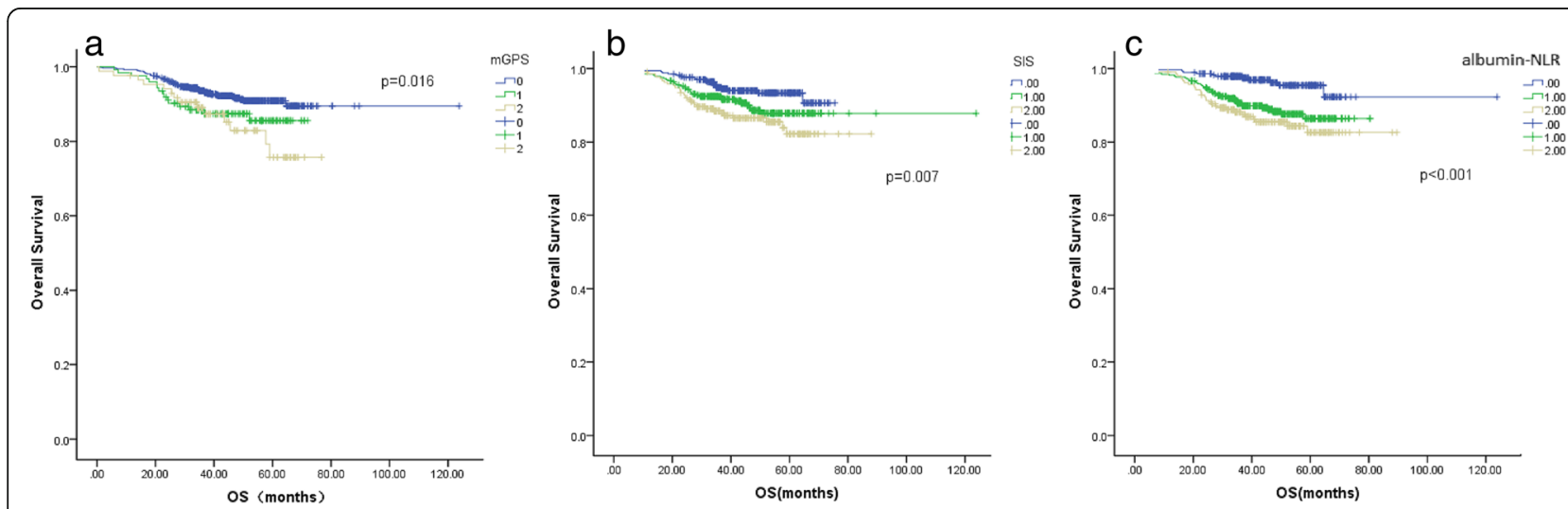

Fig. 1 Kaplan-Meier and multivariate analysis of mGPS, SIS and albumin-NLR in the group including all CRC patients. The mGPS, SIS and albuminNLR were significantly associated with OS ( $m G P S, p=0.016$; SIS, $p<0.001$; albumin-NLR, $p=0.007$ ). The lines of albumin-NLR and SIS separated very well $(\mathbf{b}, \mathbf{c})$, but overlapped each other according to mGPS (a) 


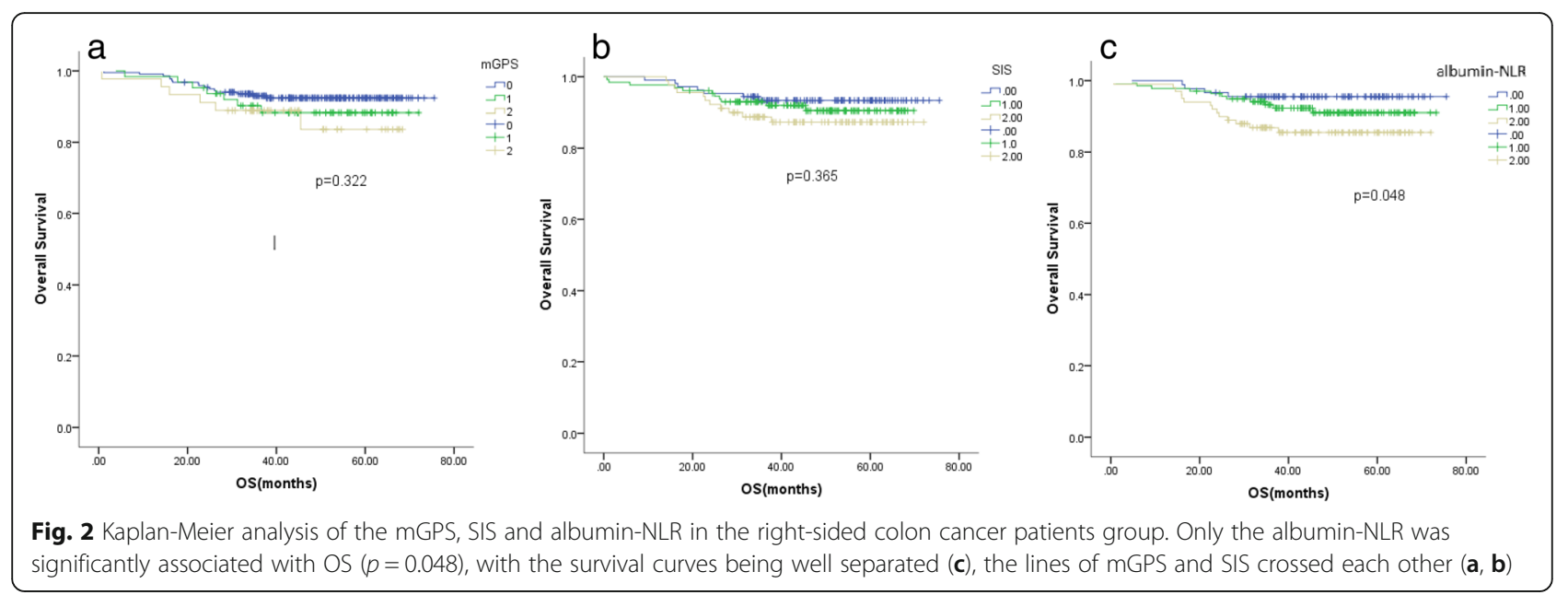

\section{Discussion}

Our research was the first study to compare the SIS, mGPS and albumin-NLR as prognostic indicators for OS in CRC patients with radical resection. We demonstrated that the albumin-NLR was a more powerful prognostic factor for OS than the other two. In addition, we also analysed the relationship between inflammation-based scores and local inflammation indices, which was an important supplement to inflammation-based prognostic indices.

With the emergence of immunotherapy, the immune system status and inflammation severity have become the focus of many studies. The traditional TNM stage is limited in this regard, indicating the need for a more robust prognostic system. Inflammation factors can quantify and characterize the inflammatory state of the patient and the general immune system status.

The NLR has been thoroughly studied and identified as an independent prognostic factor for OS in multiple solid tumour sites [21], including CRC [8]. Several studies have demonstrated that the NLR was the only independent prognostic factor for OS in CRC patients among the LMR, platelet-to-lymphocyte ratio (PLR) and the prognostic nutritional index (PNI) $[9,20]$. However, the LMR has been shown to be a valuable prognostic factor for OS in several solid tumour types [22-24]. Previously, Chan et al. demonstrated that the LMR was superior to both the NLR and PLR as a predictor of OS in resectable CRC patients [10]. The mGPS criterion is a well-known inflammation index for OS in CRC $[13,25]$. However, Suzuki et al. recently discovered the SIS to be superior to the mGPS in CRC patients who received curative surgical resection [14]. At present, there is no consensus as to which inflammatory biomarker is the most clinically useful or the best predictor of prognosis for CRC.

The mGPS and SIS are composed of a CRP and a simple inflammation factor. As the NLR is a well-known inflammation factor for CRC patients and is also compared to the LMR, we constructed the albumin-NLR by incorporating CRP and the NLR.
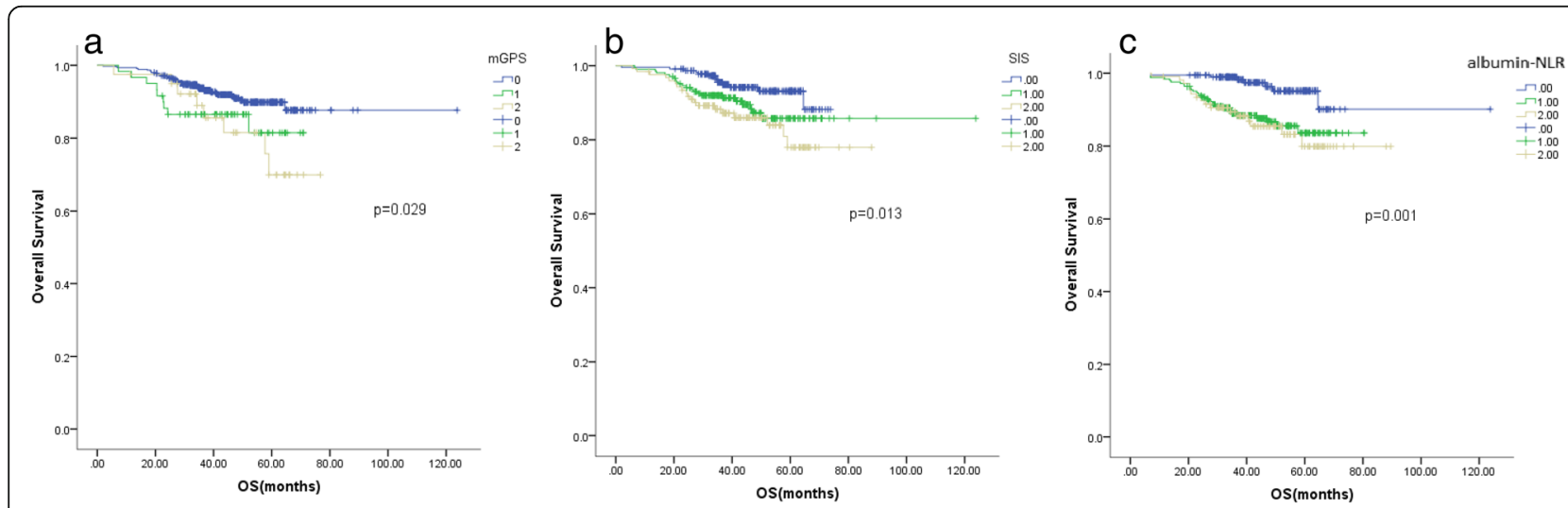

Fig. 3 Kaplan-Meier analysis of the mGPS, SIS and albumin-NLR in the group of patients with left-sided colon cancer. All three score types were independent factors for OS ( $m G P S, p=0.029$; SIS $p=0.0013$; albumin-NLR, $p=0.001$ ). The lines scored 0 seperated with the lines scored 1 and 2 in mGPS, SIS, albumin-NLR; but, the lines scored 1 overlaped the lines scored 2 in $\mathrm{mGPS}$, SIS and albumin-NLR (a, b, c) 


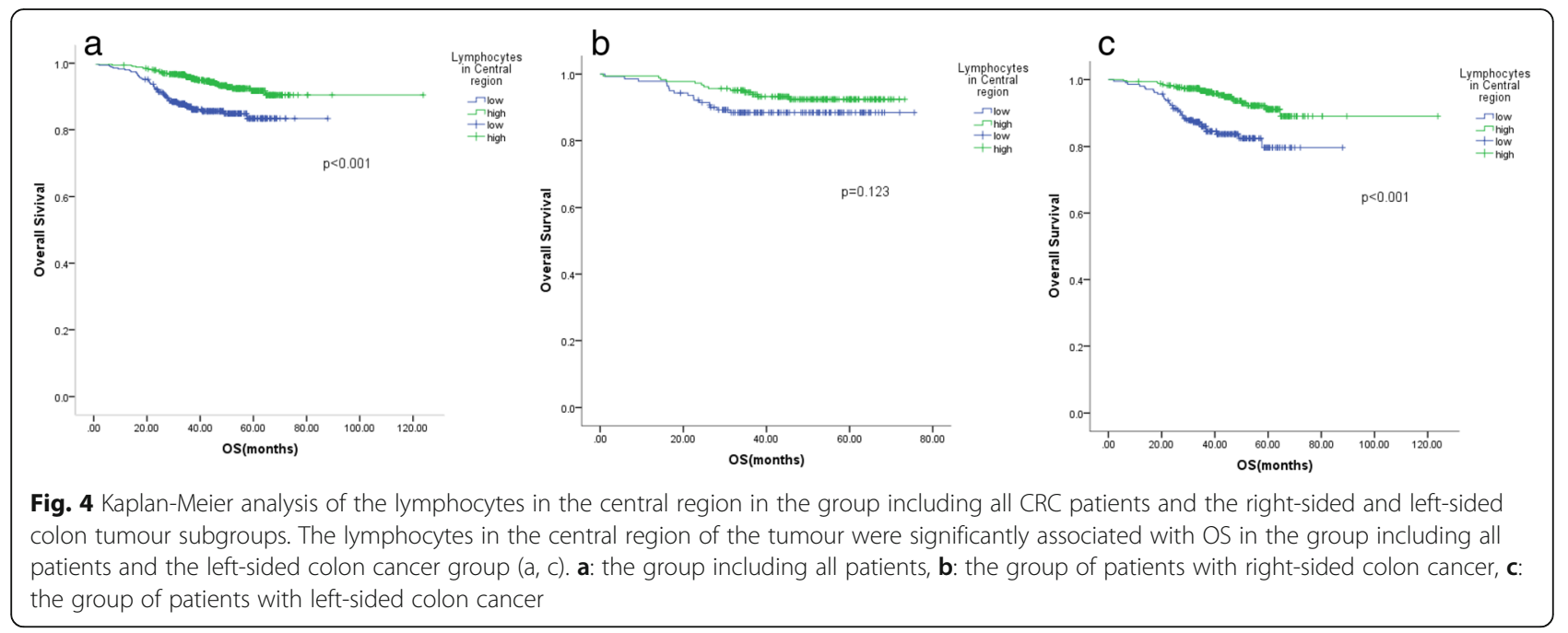

In our study, the albumin-NLR showed the optimal Kaplan-Meier curves in the group including all CRC patients, and it was also the only significant marker for OS in the right-sided colon cancer subgroup. In the left-sided CRC subgroup, the three inflammation factors were almost equally robust in the Kaplan-Meier analysis. The albuminNLR proved to be the only independent prognostic index for OS by the multivariate Cox proportional-hazard regression analysis. Furthermore, the time-dependent ROC curve showed that the albumin-NLR was continually superior to the mGPS and SIS in the three patient groups in our study. The AUC of the albumin-NLR for OS ranged from 0.5252 to 0.877 and ranged from 0.602 to 0.803 in the group including all patients and the left-sided colon group, respectively. In the right-sided colon group, the time-dependent ROC curve of the albumin-NLR was superior to the mGPS and SIS starting at the 16th month after surgery, and the AUC of the albumin-NLR for OS ranged from 0.579 to 0.669 since then. Although the AUC of the albumin-NLR in our study was less than 0.7, it was in accordance with the AUC of the classic prognostic factors reported for CRC patients, such as CEA [26, 27].

The indices derived from the comprehensive blood tests are a reflection of the inflammation status generated both at the local level and systemically before resection. It has been reported that tumour-infiltrating lymphocytes and neutrophils correlated with peripheral blood lymphocytes and neutrophils [17]. The local tumour-infiltrating inflammatory cells correlated to the patient outcomes in our study. The patients with high neutrophil density in the central region of the tumour and the tumour invasive margin correlated with higher albumin-NLR and SIS score groups. Patients with lower lymphocyte counts in the tumour invasive margin showed a higher albumin-NLR score, but the other two inflammatory markers showed no relationship. Patients without local Crohn's-like reactions had higher albumin-NLR and SIS scores. There have been previous

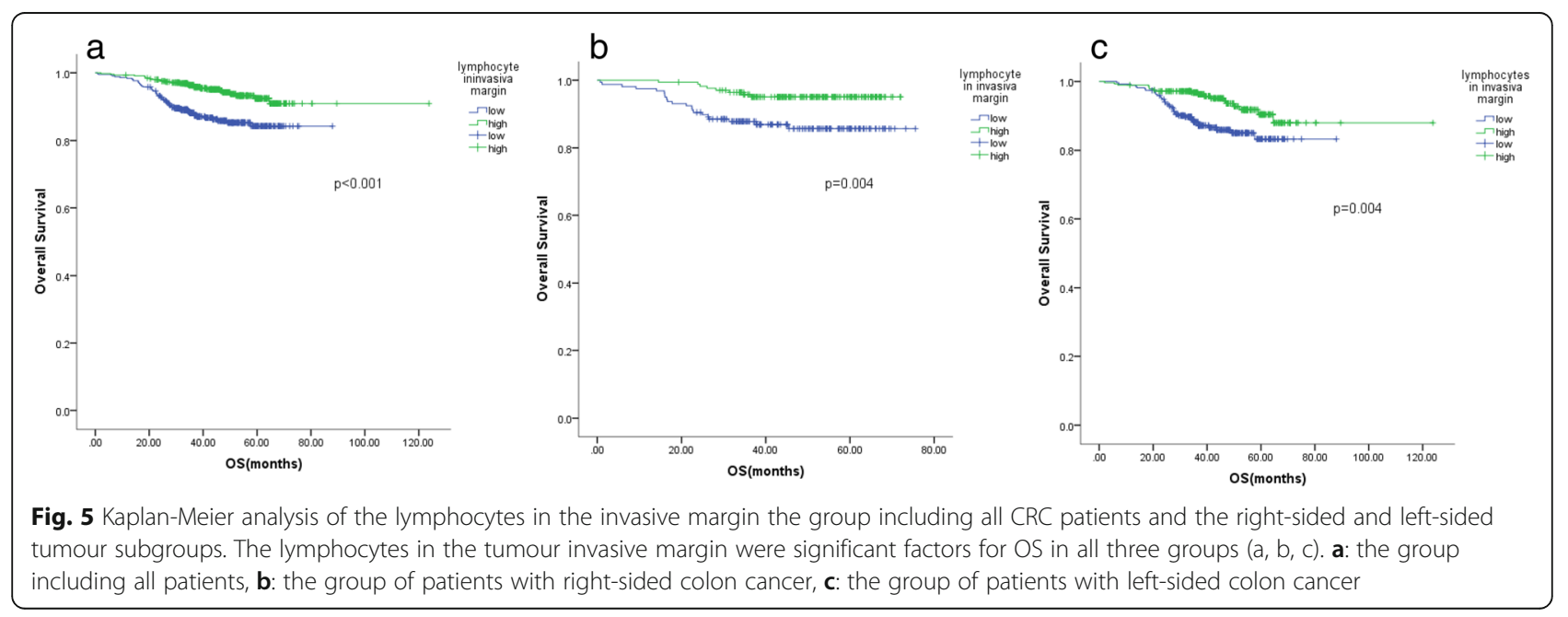


Table 2 Univariate and Multivariate Analysis of Clinicopathological Characteristics in Relation to the Overall Survival in Patients with CRC Undergoing Curative Resection

\begin{tabular}{|c|c|c|c|c|}
\hline & Univariate & & Multivariate & \\
\hline & $\mathrm{HR}(95 \% \mathrm{Cl})$ & $p$ & $\mathrm{HR}(95 \% \mathrm{Cl})$ & $p$ \\
\hline mGPS & $1.49(1.129-1.965)$ & 0.005 & $1.223(0.867-1.725)$ & 0.253 \\
\hline SIS & 1.5441.173-2.033) & 0.002 & $0.869(0.562-1.345)$ & 0.529 \\
\hline Albumin-NLR & $1.812(1.35-2.433)$ & $<0.001$ & $2.112(1.314-3.395)$ & 0.002 \\
\hline Age & $1.025(1.00701 .044)$ & 0.007 & $1.032(1.012-1.052)$ & 0.001 \\
\hline TNM stage & $2.251(1.553-3.263)$ & $<0.001$ & $0.845(0.419-1.705)$ & 0.638 \\
\hline T stage & $1.674(1.162-2.412)$ & 0.006 & $1.332(0.877-2.023)$ & 0.179 \\
\hline N stage & $1.759(1.397-2.241)$ & $<0.001$ & $1.760(1.142-2.712)$ & 0.01 \\
\hline TLN & $0.425(0.276-0.654)$ & $<0.001$ & $0.375(0.164-0.857)$ & 0.02 \\
\hline NLN & $0.383(0.248-0.591)$ & $<0.001$ & $1.050(0.460-2.397)$ & 0.908 \\
\hline Vascular invasion & $2.501(1.606-3.893)$ & $<0.001$ & $0.557(0.903-2.686)$ & 0.111 \\
\hline Peripheral nerve invasion & $1.732(1.046-2.868)$ & 0.033 & $1.169(0.674-2.025)$ & 0.578 \\
\hline Lymphocytes in invasive margin & $0.396(0.249-0.63)$ & $<0.001$ & $0.694(0.385-1.249)$ & 0.223 \\
\hline Lymphocytes in central region & $0.405(0.261-0.627$ & $<0.001$ & $0.680(0.394-1.174)$ & 0.167 \\
\hline
\end{tabular}

mGPS modified Glasgow Prognostic Score, SIS systemic inflammation score, albumin-NLR albumin- neutrophil-to-lymphocyte ratio; TLN total lymph nodes resected, $N L N$ negative lymph nodes resected, $H R$ hazard ratio, $C l$ confidence interval

reports of high neutrophil and lymphocyte counts in the central region of the tumour and the tumour invasive margin being related to longer survival [28]. Our findings also showed that patients with higher lymphocyte counts in the central region of the tumour and the tumour invasive margin were related to better patient survival. The albumin-NLR was the only inflammation score type found to predict OS, indicating that this measure may better reflect the status of the local tumour-infiltrating inflammatory cells.

The patients with MSI had higher albumin-NLR and SIS scores. CRC patients with MSI are known to be more sensitive to immunotherapy [29]. The MSI status was associated with a high mutational burden and immune infiltration [29], which provided recognizable cancer antigens for the immune system. The active immune system can be reflected by the local and systemic inflammation levels during malignancy. Moreover, inflammation indices that are derived from the peripheral blood better reflect the current immune status than other markers, such as CRP.

$\mathrm{C}$-reactive protein is an acute temporal response protein, which reflects acute trauma or the inflammatory condition of the patient, instead of the local or systemic inflammation status when cancer is present. The markers derived from the peripheral blood were more effective at this point. We also analysed the prognostic value
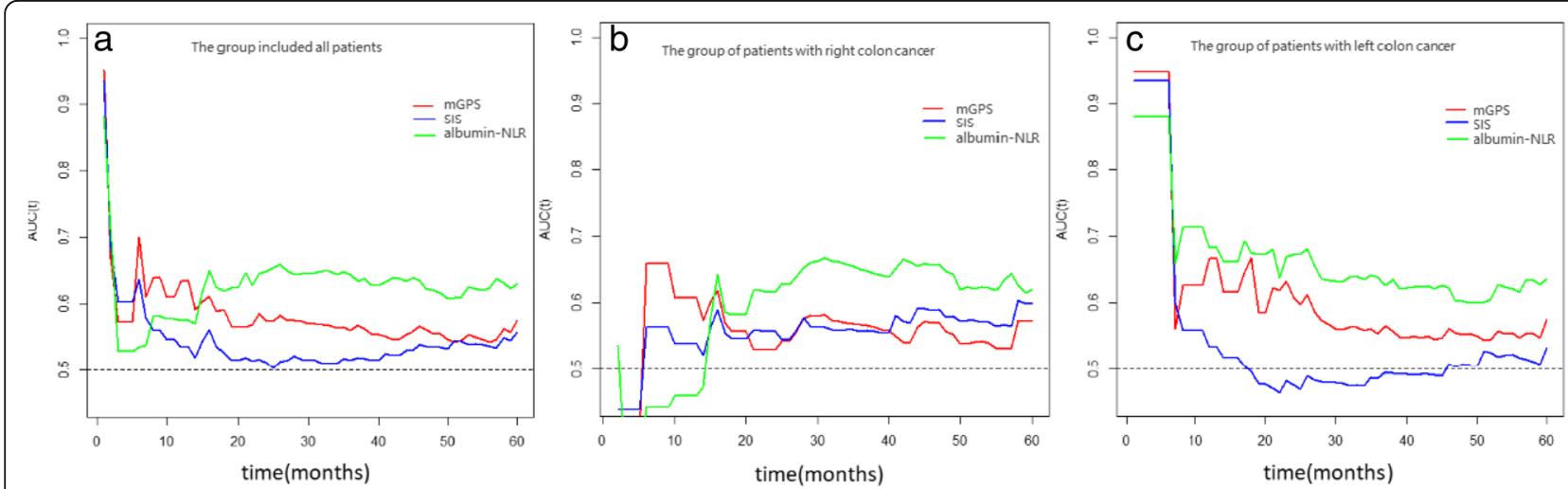

Fig. 6 Time-dependent ROC curves for the MGPS, SIS and albumin-NLR. The horizontal axis represents the year after surgery and the vertical axis represents the estimated area under the ROC curve for survival at the time of interest. Red, blue and green solid lines represent the estimated AUC of the mGPS, SIS and albumin-NLR, respectively. The albumin-NLR was superior to the mGPS and SIS in predicting the 5-year survival rate of CRC patients in all three groups. a: the group including all patients, $\mathbf{b}$ : the group of patients with right-sided colon cancer, $\mathbf{c}$ : the group of patients with left-sided colon cancer 
of the NLR and LMR for OS by the Kaplan-Meier method. The NLR was a significant factor for OS $(p=0.023)$, but the LMR failed to predict OS $(p=0.065)$. This result lends some explanation for why the albumin-NLR was superior to the SIS in terms of OS in our study.

The accumulated data suggest that inflammatory markers are associated with pathological features and prognosis in CRC patients. The pathological factors, such as the T stage, N stage, TLN, and NLN are well-known prognostic factors. For our study, we enrolled only limited stage patients, thereby requiring the analysis of the prognostic power of the three inflammation scoring methods in subgroups of the T stage, N stage, TLN, and NLN, by the Kaplan-Meier method. The albumin-NLR curve for OS was the most robust. From the results of the Kaplan-Meier analysis, it can be concluded that the albumin-NLR was a powerful prognostic factor for OS. The albumin-NLR can further predict the prognosis for the T stage, $\mathrm{N}$ stage, TLN, and NLN subgroups and should be considered as a supplement to TNM staging.

There were several limitations to this study. First, this report had a retrospective study design, which may induce some selection bias. Second, most of the study patients were administered routine adjuvant chemotherapy, but since the chemotherapy data were incomplete, we could not thoroughly analyse any possible relationship between treatment agents and inflammation factors. Moreover, the data regarding local macrophages was not available. Finally, since the albumin-NLR is a novel inflammation score, more research is needed to validate this factor's prognostic value and further applications.

\section{Conclusions}

In conclusion, the albumin-NLR inflammation scoring method outperformed both the SIS and mGPS in predicting survival in CRC patients undergoing resection, indicating that albumin-NLR is a useful inflammatory marker. Further prospective studies should be conducted to confirm these results.

\begin{abstract}
Abbreviations
AJCC: American Joint Committee on Cancer; albumin-NLR: Albuminneutrophil-to-lymphocyte ratio; CIC: Intratumoural chronic inflammatory cell; CRC: Colorectal cancer; CRP: C-reactive protein; HRs: Hazard ratios; LMR: Lymphocyte-to-monocyte ratio; mGPS: Modified Glasgow Prognostic Score; MSI: Microsatellite instability; NLN: Negative lymph nodes resected; PLR: Platelet-to-lymphocyte ratio; PNI: Prognostic nutritional index; ROC: Receiver operating characteristics analysis; SIS: Systemic inflammation score; TILs: Tumour-infiltrating lymphocytes; TLN: Total lymph nodes resected
\end{abstract}

\section{Acknowledgements}

The authors thank the patients and the colleagues in Sun Yat-sen University Cancer Center who participated in this study.

\section{Funding}

This study was supported by grants from the Natural Science Foundation of Guangdong, China (2015A030313010), Science and Technology Program of Guangzhou, China (1563000305) and National Natural Science Foundation of China (81572409).

\section{Availability of data and materials}

The data was collected by our authors at the Sun Yat-Sen University Cancer Center. The research data was registered at the Research Data Deposit of Sun Yat-Sen University Cancer Center. The RDD number for this dataset is RDDA2018000829.

\section{Authors' contributions}

Wang Fang and He Wenzhuo designed the study, performed the statistical analysis and drafted the manuscript. Jiang Chang, Guo Guifang Ke Bin and Dai Qiangsheng collected the clinical data and revised the manuscript critically. Xia Liangping and Long Jianting designed the study and critically reviewed the manuscript. All authors read and approved the final manuscript.

\section{Authors' information}

Wang Fang Oncologist, The First Affiliated Hospital of Sun Yat-sen University, Department of Oncology. 58, the 2nd Zhongshan Road, Guangzhou 510,080, China. Email: wangfang1368@126.com

He Wenzhuo Oncologist, Sun Yat-sen University Cancer Center; State Key Laboratory of Oncology in South China; Collaborative Innovation Center for Cancer Medicine. 651, Dongfengdong Road, Guangzhou 510,060, China. Email: happyhewenzhuo@163.com

Jiang Chang Oncologist, Sun Yat-sen University Cancer Center; State Key Laboratory of Oncology in South China; Collaborative Innovation Center for Cancer Medicine. 651, Dongfengdong Road, Guangzhou 510,060, China. Email: jiangchang@sysucc.org.cn

Guo Guifang Professor, Sun Yat-sen University Cancer Center; State Key Laboratory of Oncology in South China; Collaborative Innovation Center for Cancer Medicine. 651, Dongfengdong Road, Guangzhou 510,060, China. Email: gquifang@163.com

Ke Bin Physician, The First Affiliated Hospital of Sun Yat-sen University, Department of Traditional Chinese Medicine. 58, the 2nd Zhongshan Road, Guangzhou 510,080, China. Emal:jackhorn@163.com

Dai Qiangsheng Professor, The First Affiliated Hospital of Sun Yat-sen University, Department of Oncology. 58, the 2nd Zhongshan Road, Guangzhou 510,080, China. Email: daiqs@163.com

Long Jianting Professor, The First Affiliated Hospital of Sun Yat-sen University, Department of Oncology. 58, the 2nd Zhongshan Road, Guangzhou 510,080, China. Email: longjianting@163.com

Xia Liangping Professor, Sun Yat-sen University Cancer Center; State Key Laboratory of Oncology in South China; Collaborative Innovation Center for Cancer Medicine. 651, Dongfengdong Road, Guangzhou 510,060, China. Email: xialiangping@163.com

\section{Ethics approval and consent to participate}

The study design was approved by the appropriate ethics review board of Sun Yat-sen University Cancer Center (GZR2015-034). This study is a retrospective research and the data were analysed anonymously. The need for consent to participate was waived by Ethics Commission of Sun Yat-sen University Cancer Center.

\section{Consent to publication}

Not applicable.

\section{Competing interests}

The authors do not have any competing interests in the manuscript.

\section{Publisher's Note}

Springer Nature remains neutral with regard to jurisdictional claims in published maps and institutional affiliations.

\section{Author details}

${ }^{1}$ Sun Yat-sen University Cancer Center; State Key Laboratory of Oncology in South China, 651, Dongfengdong Road, Guangzhou 510060, China.

${ }^{2}$ Department of Oncology, The First Affiliated Hospital of Sun Yat-sen University, 58, the 2nd Zhongshan Road, Guangzhou 510080, China. ${ }^{3}$ Sun Yat-sen University Cancer Center; State Key Laboratory of Oncology in South China; Collaborative Innovation Center for Cancer Medicine, 651,

Dongfengdong Road, Guangzhou 510060, China. ${ }^{4}$ Department of Traditional Chinese Medicine, The First Affiliated Hospital of Sun Yat-sen University, 58, the 2nd Zhongshan Road, Guangzhou 510080, China. 
Received: 27 February 2018 Accepted: 20 September 2018

Published online: 12 November 2018

\section{References}

1. Siegel RL, Miller KD, Jemal A. Cancer statistics, 2016. CA Cancer J Clin. 2016; 66(1):7-30.

2. McArdle CS, Hole DJ. Outcome following surgery for colorectal cancer: analysis by hospital after adjustment for case-mix and deprivation. Br J Cancer. 2002;86(3):331-5.

3. Fridman WH, Pages F, Sautes-Fridman C, Galon J. The immune contexture in human tumours: impact on clinical outcome. Nat Rev Cancer. 2012;12(4): 298-306.

4. Diakos Cl, Charles KA, McMillan DC, Clarke SJ. Cancer-related inflammation and treatment effectiveness. Lancet Oncol. 2014;15(11):e493-503.

5. Grivennikov SI, Greten FR, Karin M. Immunity, inflammation, and cancer. Cell. 2010;140(6):883-99.

6. Gooden MJ, de Bock GH, Leffers N, Daemen T, Nijman HW. The prognostic influence of tumour-infiltrating lymphocytes in cancer: a systematic review with meta-analysis. Br J Cancer. 2011;105(1):93-103.

7. Mlecnik B, Tosolini M, Kirilovsky A, Berger A, Bindea G, Meatchi T, Bruneval P, Trajanoski Z, Fridman WH, Pages F, et al. Histopathologic-based prognostic factors of colorectal cancers are associated with the state of the local immune reaction. J Clin Oncol. 2011;29(6):610-8.

8. Guthrie GJ, Charles KA, Roxburgh CS, Horgan PG, McMillan DC, Clarke SJ. The systemic inflammation-based neutrophil-lymphocyte ratio: experience in patients with cancer. Crit Rev Oncol Hematol. 2013;88(1):218-30.

9. Kwon HC, Kim SH, Oh SY, Lee S, Lee JH, Choi HJ, Park KJ, Roh MS, Kim SG, Kim HJ, et al. Clinical significance of preoperative neutrophil-lymphocyte versus platelet-lymphocyte ratio in patients with operable colorectal cancer. Biomarkers. 2012;17(3):216-22.

10. Chan JC, Chan DL, Diakos Cl, Engel A, Pavlakis N, Gill A, Clarke SJ. The lymphocyte-to-monocyte ratio is a superior predictor of overall survival in comparison to established biomarkers of Resectable colorectal Cancer. Ann Surg. 2017;265(3):539-46

11. Leitch EF, Chakrabarti M, Crozier JE, McKee RF, Anderson JH, Horgan PG, McMillan DC. Comparison of the prognostic value of selected markers of the systemic inflammatory response in patients with colorectal cancer. $\mathrm{Br}$ Cancer. 2007;97(9):1266-70.

12. Ishizuka M, Nagata H, Takagi K, Horie T, Kubota K. Inflammation-based prognostic score is a novel predictor of postoperative outcome in patients with colorectal cancer. Ann Surg. 2007:246(6):1047-51.

13. Park JH, Watt DG, Roxburgh CS, Horgan PG, McMillan DC. Colorectal Cancer, systemic inflammation, and outcome: staging the tumor and staging the host. Ann Surg. 2016;263(2):326-36.

14. Suzuki Y, Okabayashi K, Hasegawa H, Tsuruta M, Shigeta K, Kondo T, Kitagawa Y. Comparison of preoperative inflammation-based prognostic scores in patients with colorectal Cancer. Ann Surg. 2016.

15. Sato E, Olson SH, Ahn J, Bundy B, Nishikawa H, Qian F, Jungbluth AA, Frosina D, Gnjatic S, Ambrosone C, et al. Intraepithelial CD8+ tumor-infiltrating lymphocytes and a high $\mathrm{CD} 8+/$ regulatory $T$ cell ratio are associated with favorable prognosis in ovarian cancer. Proc Natl Acad Sci U S A. 2005;102(51):18538-43.

16. Tomsova M, Melichar B, Sedlakova I, Steiner I. Prognostic significance of CD3 + tumor-infiltrating lymphocytes in ovarian carcinoma. Gynecol Oncol. 2008; 108(2):415-20

17. Turner N, Wong HL, Templeton A, Tripathy S, Whiti Rogers T, Croxford M, Jones I, Sinnathamby M, Desai J, Tie J, et al. Analysis of local chronic inflammatory cell infiltrate combined with systemic inflammation improves prognostication in stage $\|$ colon cancer independent of standard clinicopathologic criteria. Int J Cancer. 2016;138(3):671-8.

18. Mei Z, Liu Y, Liu C, Cui A, Liang Z, Wang G, Peng H, Cui L, Li C. Tumourinfiltrating inflammation and prognosis in colorectal cancer: systematic review and meta-analysis. Br J Cancer. 2014;110(6):1595-605.

19. Jass JR. Lymphocytic infiltration and survival in rectal cancer. J Clin Pathol. 1986;39(6):585-9.

20. Guthrie GJ, Roxburgh CS, Farhan-Alanie OM, Horgan PG, McMillan DC. Comparison of the prognostic value of longitudinal measurements of systemic inflammation in patients undergoing curative resection of colorectal cancer. Br J Cancer. 2013;109(1):24-8.

21. Templeton AJ, McNamara MG, Seruga B, Vera-Badillo FE, Aneja P, Ocana A Leibowitz-Amit R, Sonpavde G, Knox JJ, Tran B, et al. Prognostic role of neutrophil-to-lymphocyte ratio in solid tumors: a systematic review and meta-analysis. J Natl Cancer Inst. 2014;106(6):dju124.

22. Stotz M, Pichler M, Absenger G, Szkandera J, Arminger F, Schaberl-Moser R, Samonigg H, Stojakovic T, Gerger A. The preoperative lymphocyte to monocyte ratio predicts clinical outcome in patients with stage III colon cancer. Br J Cancer. 2014;110(2):435-40.

23. Nishijima TF, Muss HB, Shachar SS, Moschos SJ. Comparison of efficacy of immune checkpoint inhibitors (ICls) between younger and older patients: a systematic review and meta-analysis. Cancer Treat Rev. 2016;45:30-7.

24. Shibutani M, Maeda K, Nagahara H, Ohtani H, Sakurai K, Yamazoe S, Kimura $\mathrm{K}$, Toyokawa $\mathrm{T}$, Amano $\mathrm{R}$, Tanaka $\mathrm{H}$, et al. Prognostic significance of the lymphocyte-to-monocyte ratio in patients with metastatic colorectal cancer World J Gastroenterol. 2015;21(34):9966-73.

25. Richards $\mathrm{CH}$, Leitch EF, Horgan PG, Anderson JH, McKee RF, McMillan DC. The relationship between patient physiology, the systemic inflammatory response and survival in patients undergoing curative resection of colorectal cancer. Br J Cancer. 2010;103(9):1356-61.

26. Yang $L, G e L Y, Y u T$, Liang $Y$, Yin $Y$, Chen $H$. The prognostic impact of serum bilirubin in stage IV colorectal cancer patients. J Clin Lab Anal. 2018;32(2): e22272.

27. Zhan X, Sun X. Combined Detection of Preoperative Neutrophil-toLymphocyte Ratio and CEA as an Independent Prognostic Factor in Nonmetastatic Patients Undergoing Colorectal Cancer Resection Is Superior to NLR or CEA Alone, vol. 2017; 2017. p. 3809464.

28. Klintrup K, Makinen JM, Kauppila S, Vare PO, Melkko J, Tuominen H, Tuppurainen K, Makela J, Karttunen TJ, Makinen MJ. Inflammation and prognosis in colorectal cancer. Eur J Cancer (Oxford, England: 1990). 2005; 41(17):2645

29. Lal N, Beggs AD, Willcox BE, Middleton GW. An immunogenomic stratification of colorectal cancer: implications for development of targeted immunotherapy. Oncoimmunology. 2015;4(3):e976052.

\section{Ready to submit your research? Choose BMC and benefit from:}

- fast, convenient online submission

- thorough peer review by experienced researchers in your field

- rapid publication on acceptance

- support for research data, including large and complex data types

- gold Open Access which fosters wider collaboration and increased citations

- maximum visibility for your research: over $100 \mathrm{M}$ website views per year

At BMC, research is always in progress.

Learn more biomedcentral.com/submissions 